\title{
Coulomb breakup of light composite nuclei
}

\author{
J.A. Tostevin, S. Rugmai and R.C. Johnson, ${ }^{1}$ H. Okamura, S. Ishida $[*]$, N. Sakamoto[*], \\ H. Otsu[*], T. Uesaka[*], T. Wakasa[*] and H. Sakai, ${ }^{2}$ T. Niizeki, H. Toyokawa[*], \\ Y. Tajima[†] and H. Ohnuma $[\ddagger],{ }^{3}$ M. Yosoi, ${ }^{4}$ K. Hatanaka, ${ }^{5}$ and T. Ichihara ${ }^{6}$ \\ ${ }^{1}$ Department of Physics, University of Surrey, Guildford, Surrey, GU2 5XH, \\ United Kingdom \\ ${ }^{2}$ Department of Physics, University of Tokyo, Bunkyo-ku, Tokyo 113, Japan \\ ${ }^{3}$ Department of Physics, Tokyo Institute of Technology, Meguro-ku, Tokyo 152, Japan \\ ${ }^{4}$ Department of Physics, Kyoto University, Sakyo-ku, Kyoto 606, Japan \\ ${ }^{5}$ Research Center for Nuclear Physics, \\ Osaka University, Ibaraki, Osaka 567, Japan \\ ${ }^{6}$ The Institute of Physical and Chemical Research (RIKEN), Saitama 351-01, Japan
}

(Dated: November 5, 2011)

\begin{abstract}
We present a new analysis of the Coulomb dissociation of nuclei comprising a charged core and one or more neutral valence particles at energies of tens of $\mathrm{MeV} /$ nucleon and above. The approach assumes only that excitation of the projectile is to the low energy continuum. A successful comparison is made with new high precision measurements of small angle deuteron dissociation at $140 \mathrm{MeV}$ incident energy.
\end{abstract}

PACS numbers: 
Studies of Coulomb breakup of composite nuclei have been of constant interest in nuclear physics and in applications to nuclear astrophysics. The recent discovery [5] of very extended halo nuclei with a dense charged core and one, or sometimes two, very loosely bound valence neutrons, has renewed interest in methods for the calculation of Coulomb dissociation. This is a critical reaction channel in the scattering of such nuclei from a stable target. While these exotic nuclei are an exciting application, the low intensities currently available of secondary beams of these new nuclear species means that present data carry significant experimental uncertainty, are often inclusive data, and are still quite limited. Inevitably these data do not yet challenge approximate theoretical descriptions effectively. In particular, available data cannot yet compete with those from high intensity ion-sources and light-ion induced reactions.

In this Letter we reconsider calculations of Coulomb dissociation based on a theory which makes the single approximation that breakup is to the low energy continuum. The theory is fully quantum mechanical and is non-perturbative. It is shown to lead to a breakup amplitude of similar form to that obtained earlier, using approximations to distorted waves Born approximation (DWBA) theory, but which differs significantly from these in both detail and in interpretation. Without the DWBA weak coupling approximation we are able to calculate explicitly the post-form of the quantum mechanical breakup transition amplitude and make comparisons with the most exclusive experimental data available.

The theory presented thus offers an alternative scheme to semi-classical descriptions of the excitation process, whether based on coupled channels, e.g [6], or direct time-dependent solution methods, e.g. [7]. There are however no restrictions on Coulomb multipole excitations, on the order of excitation corrections, no zero-range approximations to interactions $[8,9]$, or reference or approximations made to assumed semi-classical trajectories. A crucial requirement for our analysis, however, is that the Coulomb interaction acts only on a single charged fragment in the composite projectile. The method is therefore not applicable to many very interesting situations, such as the Coulomb dissociation of ${ }^{8} \mathrm{~B}$, for which semi-classical methods have been extended to consider higher order excitation and multipole effects [10].

The theory is compared here with recent precise, kinematically complete, deuteron breakup data into very forward angles [11], a system for which there are no ambiguities associated with the projectile structure. Strong indications of the importance of dipole 
Coulomb breakup for such a restricted detection geometry, manifest as a characteristic double peaking in the measured triple differential cross section as a function of detected proton energy, have been reported previously [12]. Applications of the current theory to the breakup of halo nuclei will be discussed elsewhere.

We consider here the Coulomb dissociation of a two-body projectile nucleus by a target of mass $m_{t}$ and charge $Z_{t}$. The projectile is assumed to consist of a charged core of mass $m_{c}$ and charge $Z_{c}$ and a neutral valence particle of mass $m_{v}$, bound by a potential $V_{v c}$. With the application to the deuteron in mind then the projectile has only one bound state. We adopt the coordinate system shown in Figure 1. Thus we assume a (Coulomb) interaction $V_{c t}$ between the core and target nuclei only. The equation satisfied by the three-body scattering wave function $\Psi_{\mathbf{k}_{p}}^{(+)}(\mathbf{r}, \mathbf{R})$, corresponding to the projectile incident with momentum $\mathbf{k}_{p}$ in the center of mass (c.m.) frame, is therefore

$$
\left[T_{\mathbf{R}}+V_{c t}\left(\mathbf{R}-\gamma_{v c} \mathbf{r}\right)+H_{v c}-E\right] \Psi_{\mathbf{k}_{p}}^{(+)}(\mathbf{r}, \mathbf{R})=0
$$

Here $H_{v c}$ is the internal Hamiltonian for the valence-core system, $\mathbf{R}$ is the projectile-target separation, $T_{\mathbf{R}}$ the corresponding kinetic energy operator, and $\mathbf{r}$ is the core to valence particle relative coordinate. The quantity $\gamma_{v c}=m_{v} /\left(m_{v}+m_{c}\right)$, so $\mathbf{R}-\gamma_{v c} \mathbf{r}=\mathbf{R}_{c}$ is the targetcore separation. The wave function $\Psi_{\mathbf{k}_{p}}^{(+)}(\mathbf{r}, \mathbf{R})$ therefore satisfies the scattering boundary conditions

$$
\Psi_{\mathbf{k}_{p}}^{(+)}(\mathbf{r}, \mathbf{R})=\phi_{0}(\mathbf{r}) e^{i \mathbf{k}_{p} \cdot \mathbf{R}}+\text { outgoing waves },
$$

where, for a projectile with a single bound state, the outgoing waves include only elastic and elastic break-up channels. The projectile ground state wavefunction $\phi_{0}(\mathbf{r})$ satisfies $H_{v c} \phi_{0}(\mathbf{r})=-\varepsilon_{0} \phi_{0}(\mathbf{r})$. While, strictly, the incident plane wave boundary condition stated in Eq. (2) is incorrect in the presence of Coulomb interactions, our final formulae can be justified by considering the limit of an appropriately screened Coulomb problem.

The essential step in the present analysis is to assume that the breakup configurations strongly coupled in the three-body equation (1) have core-valence particle relative energies $\varepsilon \ll E$, the incident energy. The actual spectrum of breakup states excited will be dictated by the properties of $V_{c t}$, in this case the Coulomb interaction of the core and target. The long range of this interaction means that it may act repeatedly, to high order, but, given its slow spatial variation, matrix elements of $V_{c t}$ will favour coupling to states in close proximity in energy. Assuming $\varepsilon \ll E$, little error will be expected upon replacing $H_{v c}$ in Eq. (1) 
by a representative constant energy. Moreover, if this constant is chosen as $-\varepsilon_{0}$, we will guarantee that the solution of the resulting approximate three-body equation satisfies the correct incident wave boundary conditions and that the dominant elastic channel has the correct channel energy. In this physical limit, then

$$
\left[T_{\mathbf{R}}+V_{c t}\left(\mathbf{R}-\gamma_{v c} \mathbf{r}\right)-E_{0}\right] \bar{\Psi}_{\mathbf{k}_{p}}^{(+)}(\mathbf{r}, \mathbf{R})=0
$$

where $\mathbf{r}$ enters simply as a parameter, $E_{0}=E+\varepsilon_{0}=\hbar^{2} k_{p}^{2} / 2 \mu$ is the incident c.m. kinetic energy and $\mu$ is the projectile-target reduced mass. This replacement of $H_{v c}$ by $-\varepsilon_{0}$, commonly referred to as the adiabatic [13] or sudden [14] approximation, assumes the continuum spectrum of $H_{v c}$ is degenerate with the ground state.

The now parametric dependence of Eq. (3) on $\mathbf{r}$, and the invariance of the kinetic energy operator under spatial translations, means that the most general solution $\bar{\Psi}_{\mathbf{k}_{p}}$ of this approximate three-body equation takes the form of the product of a function $\mathcal{F}(\mathbf{r})$ of the core-valence particle separation and a projectile distorted wave $\chi_{\mathbf{k}_{p}}^{(+)}$, but where the latter is evaluated at the core position coordinate $\mathbf{R}_{c}$. Explicitly,

$$
\bar{\Psi}_{\mathbf{k}_{p}}^{(+)}(\mathbf{r}, \mathbf{R})=\mathcal{F}(\mathbf{r}) \chi_{\mathbf{k}_{p}}^{(+)}\left(\mathbf{R}_{c}\right)
$$

where $\chi_{\mathbf{k}_{p}}^{(+)}$satisfies the Schrödinger equation

$$
\left[T_{\mathbf{R}}+V_{c t}(\mathbf{R})-E_{0}\right] \chi_{\mathbf{k}_{p}}^{(+)}(\mathbf{R})=0
$$

In the present context $\chi_{\mathbf{k}_{p}}^{(+)}$is a three-dimensional Coulomb distorted wave and describes the scattering of the projectile of mass $\mu$, considered pointlike, by the Coulomb interaction $V_{c t}$. The as yet unspecified multiplicative function $\mathcal{F}(\mathbf{r})$ must now be chosen so that the particular solution satisfies the required incident boundary condition Eq. (2) as well as Eq. (3). Consideration of the incident boundary condition satisfied by $\chi_{\mathbf{k}_{p}}^{(+)}\left(\mathbf{R}_{c}\right)$ and $\bar{\Psi}_{\mathbf{k}_{p}}^{(+)}(\mathbf{r}, \mathbf{R})$, Eq. (2), shows that the required, and exact, solution of Eq. (3) is [15]

$$
\bar{\Psi}_{\mathbf{k}_{p}}^{(+)}(\mathbf{r}, \mathbf{R})=\phi_{0}(\mathbf{r}) \exp \left(i \gamma_{v c} \mathbf{k}_{p} \cdot \mathbf{r}\right) \chi_{\mathbf{k}_{p}}^{(+)}\left(\mathbf{R}_{c}\right)
$$

This three-body wave function retains break-up components through its complex dependence on $\mathbf{r}$, in $\chi_{\mathbf{k}_{p}}^{(+)}$and the exponential factor $\exp \left(i \gamma_{v c} \mathbf{k}_{p} \cdot \mathbf{r}\right)$.

To use the three-body wave function of Eq. (6) to calculate a Coulomb breakup amplitude we must consider its possible limitation to certain regions of the six-dimensional $\mathbf{r}$ and $\mathbf{R}$ 
space. In particular, the very explicit form of the solution in this case makes clear that at large core-valence particle separations, $\mathbf{r} \rightarrow \infty$, the presence of $\phi_{0}$ means that $\bar{\Psi}_{\mathbf{k}_{p}}$ vanishes at least exponentially in a region which includes some breakup channels. Thus for large values of $|\mathbf{r}|$, in any direction, $\bar{\Psi}_{\mathbf{k}_{p}}^{(+)} \rightarrow 0$ exponentially and is therefore certainly inaccurate.

Our approximation to the Coulomb breakup transition amplitude, from projectile initial state $\mathbf{k}_{p}$ to a final state with core momentum $\mathbf{k}_{c}$ and valence particle momentum $\mathbf{k}_{v}$ in the c.m. frame, is therefore to use $\bar{\Psi}_{\mathbf{k}_{p}}$ as an approximation to $\Psi_{\mathbf{k}_{p}}^{(+)}$in the exact (post-form) breakup amplitude

$$
T_{\mathrm{bu}}\left(\mathbf{k}_{v} \mathbf{k}_{c}, \mathbf{k}_{p}\right)=\int d \mathbf{R} \int d \mathbf{r} \exp \left(-i \mathbf{k}_{v} \cdot \mathbf{R}_{v}\right) \chi_{\mathbf{k}_{c}}^{(-) *}\left(\mathbf{R}_{c}\right) V_{v c}(\mathbf{r}) \Psi_{\mathbf{k}_{p}}^{(+)}(\mathbf{r}, \mathbf{R})
$$

where $\chi_{\mathbf{k}_{c}}^{(-)}$is an ingoing waves Coulomb distorted wave for the core fragment. Due to $V_{v c}$ the integrals in Eq. (7) involve only small $|\mathbf{r}|$ and do not require the three-body wavefunction in regions where the approximate wavefunction may be inaccurate. Substituting for $\bar{\Psi}_{\mathbf{k}_{p}}$, making a change of integration variable from $\mathbf{R}$ to $\mathbf{R}_{c}$ and noting that $\mathbf{R}_{v}=\gamma_{t c} \mathbf{R}_{c}+\mathbf{r}$, with $\gamma_{t c}=m_{t} /\left(m_{c}+m_{t}\right)$, the breakup amplitude is seen to factorise exactly as

$$
T_{\mathrm{bu}}\left(\mathbf{k}_{v} \mathbf{k}_{c}, \mathbf{k}_{p}\right)=\left\langle\mathbf{P}_{v}\left|V_{v c}\right| \phi_{0}\right\rangle\left\langle\mathbf{Q}_{v}, \chi_{\mathbf{k}_{c}}^{(-)} \mid \chi_{\mathbf{k}_{p}}^{(+)}\right\rangle
$$

where $\mathbf{P}_{v}=\mathbf{k}_{v}-\gamma_{v c} \mathbf{k}_{p}$ and $\mathbf{Q}_{v}=\gamma_{t c} \mathbf{k}_{v}$. This is our key result.

The overlap of the three continuum functions $\left\langle\mathbf{Q}_{v}, \chi_{\mathbf{k}_{c}}^{(-)} \mid \chi_{\mathbf{k}_{p}}^{(+)}\right\rangle$which arises can be evaluated in closed form in terms of the bremsstrahlung integral $[16,17]$. The projectile vertex function $\left\langle\mathbf{P}_{v}\left|V_{v c}\right| \phi_{0}\right\rangle$ is also simply evaluated given a structure model for the projectile. Therefore, without further approximations, Eq. (8) includes a fully finite-range treatment of the core-valence particle interaction $V_{v c}$, is thus applicable to projectiles of any ground state orbital angular momentum structure, and includes breakup contributions from all Coulomb multipoles and relative orbital angular momenta between the valence and core fragments. This amplitude differs formally from those of DWBA theories since it includes the initial and final state interactions $V_{c t}$ and $V_{v c}$ to all orders.

In the following we compare the predictions of calculations made using Eq. (8) with new high precision measurements of deuteron dissociation at very forward angles. In addition to their precision, these deuteron data are free from any structure ambiguities associated with the projectile vertex $\left\langle\mathbf{P}_{v}\left|V_{v c}\right| \phi_{0}\right\rangle$. We adopt the finite range Hulthén description presented explicitly in [16], given which, the theoretical calculations are entirely parameter free. 
The $(d, p n)$ elastic breakup has been measured at the RIKEN Accelerator Research Facility in a kinematical condition of $\theta_{p}=\theta_{n}=0^{\circ}$. A deuteron beam of $140 \mathrm{MeV}$, provided by the ring cyclotron, was used to bombard the targets, ${ }^{12} \mathrm{C},{ }^{28} \mathrm{Si},{ }^{40} \mathrm{Ca},{ }^{90} \mathrm{Zr},{ }^{118} \mathrm{Sn},{ }^{165} \mathrm{Ho}$ and ${ }^{208} \mathrm{~Pb}$, which were all self-supporting foils of natural or isotopically enriched elements. Protons emitted were momentum-analyzed by using the spectrograph SMART [18] and measured by a pair of multi-wire drift chambers followed by a plastic scintillator hodoscope. Neutrons were detected by a pair of NE213 liquid scintillation counters at a distance of $12 \mathrm{~m}$ from the target and the energy was determined by the time-of-flight method. The resolution of total energy $E_{p}+E_{n}$ was $2.2 \mathrm{MeV}$ (FWHM) or better depending on the target.

The solid angles of the neutron and proton counters were $\Delta \Omega_{n}=0.45 \mathrm{msr}$ (circular shape) and $\Delta \Omega_{p}=7.2 \mathrm{msr}\left(60 \times 120 \mathrm{mrad}^{2}\right.$ rectangular shape $)$, respectively. The incident beam, having twice the magnetic rigidity as that of the protons measured, was stopped inside the dipole magnet of the spectrograph. The yoke of the dipole magnet thus served as a shield, allowing the low-background measurement. It should be noted that the situation is very different for measurements at angles other than zero degrees, where the incident beam has to be stopped inside the scattering chamber and it is then difficult to shield the background neutrons produced near the target. The neutron detection efficiency was calibrated using the ${ }^{7} \operatorname{Li}(\mathrm{p}, \mathrm{n})^{7} \mathrm{Be}$ reaction at $E_{p}=70 \mathrm{MeV}$ [19]. The proton beam was obtained by accelerating $\mathrm{H}_{2}^{+}$, the singly charged hydrogen molecule, with the same cyclotron field as that for the deuteron in order to minimize the time lag between the calibration and the breakup measurement. The energy dependence of the detection efficiency was extrapolated using a Monte-Carlo calculation. The systematic error, including those arising from uncertainties of the beam intensity and the target thickness, is estimated to be $30 \%$.

The calculations and data are compared in Figure 2 for all measured targets. The errors shown in the figure are statistical only. The solid lines show the calculations of the elastic breakup cross section as a function of detected (laboratory) proton energy calculated from the triple differential cross section $d^{3} \sigma / d \Omega_{n} d \Omega_{p} d E_{p}$ due to the finite range amplitude of Eq. (8). The appropriate three-body final state kinematics and phase space formulae for comparison with the measurements, which are presented in the laboratory frame, are presented in [20] and discussed critically in [21]. The angular dependence of these triple differential cross sections near zero degrees is rapid and qualitatively different for light and heavy targets. It was essential to integrate and then average the theoretical angular distributions 
over the specified experimental solid angles before comparison with the data. Integration of $d^{3} \sigma / d \Omega_{n} d \Omega_{p} d E_{p}$ over $\Delta \Omega_{p}$ and $\Delta \Omega_{n}$ was carried out using numerical quadratures.

The overall agreement of the calculated magnitudes, $Z_{t}$-dependence, and the proton energy dependence, with the data is good and improves with increasing target charge. This is in contrast to the results of a DWBA analysis, of the published $56 \mathrm{MeV}$ forward angle $(d, p n)$ data [12], by Samanta et al. [22]. They reported Coulomb breakup cross sections which significantly overpredicted those data and concluded that nuclear breakup contributions were large. It is not clear however that the necessary averages over the experimental acceptances, about zero degrees, were carried out in that work. Our results are consistent with an underlying picture in which Coulomb breakup is the dominant mechanism with an indication of a missing residual interference on the lighter targets, most probably resulting from smaller nuclear breakup contributions. We note that the present method is not immediately applicable to the treatment of breakup due to the nuclear components of the core and valence particle-target interactions, $V_{c t}^{N}$ and $V_{v t}^{N}$, particularly for cases, such as the deuteron, where $V_{c t}^{N} \approx V_{v t}^{N} \neq 0$. These nuclear breakup effects are being considered and will be discussed elsewhere.

Expressions with the factorised structure of our Eq. (8) have been presented previously, e.g. $[16,23]$. However these were formulated, not as above, but by starting from the weak breakup coupling DWBA approximation to the breakup amplitude, obtained by replacing $\Psi_{\mathbf{k}_{p}}^{(+)}(\mathbf{r}, \mathbf{R}) \approx \phi_{0}(\mathbf{r}) \chi_{\mathbf{k}_{p}}^{(+)}(\mathbf{R})$ in Eq. (7). For a realistic finite ranged $V_{v c}$ the resulting DWBA amplitude has itself not been evaluated exactly and therefore further approximations have been applied. These additional approximations [16, 23] are of uncertain validity, particularly at the energies under discussion here. Importantly however, these DWBA based formulations also imply a first order treatment of breakup, whereas our formulation has not used Born approximation considerations at any stage.

In Coulomb breakup the fact that the Coulomb interaction acts at the charged core and not at the centre of mass of the projectile is absolutely critical and requires careful consideration of the deviations of $\mathbf{R}_{c}$ from $\mathbf{R}$. Our formulation retains these finite $\mathbf{r}$ effects explicitly with the result that the projectile vertex $\left\langle\mathbf{P}_{v}\left|V_{v c}\right| \phi_{0}\right\rangle$ appears evaluated at $\mathbf{P}_{v}=$ $\mathbf{k}_{v}-\gamma_{v c} \mathbf{k}_{p}$, where $\mathbf{k}_{v}$ and $\mathbf{k}_{p}$ are the asymptotic outgoing valence particle and incident projectile momenta. Since $\gamma_{v c} \mathbf{k}_{p}$ is the fraction of the projectile incident momentum carried by the valence particle and, in Coulomb dissociation, momentum is transferred to the valence 
particle only by virtue of its interaction $V_{v c}$ with the core, this vertex factor displays explicitly this momentum transfer from the ground state via $V_{v c}$. The presence of the $\mathbf{k}_{p}$ term here results in $\left|\mathbf{P}_{v}\right|$ taking on small values with the result that only low momentum components of the vertex are probed. In the deuteron calculations above $\left|\mathbf{P}_{v}\right|=\left|\mathbf{k}_{n}-\frac{1}{2} \mathbf{k}_{d}\right| \approx 0.1 \mathrm{fm}^{-1}$ at the cross section maxima. This general feature will remain in applications to heavier projectile systems. It follows that, starting from our Eq. (8), a good first approximation, for bound S-state projectiles, is to replace this vertex function by its zero-range strength constant, although there is no need to do so.

We have considered the Coulomb dissociation of a composite projectile comprising a charged core and a neutral valence particle. We have shown that the use of a single approximation, that breakup is to the low energy continuum, leads to a simple and transparent expression for the breakup amplitude in the limit that strong interaction effects are neglected. The treatment allows a fully finite range treatment of the projectile vertex and does not make the weak coupling approximation of the distorted waves Born approximation. We compare the parameter free theoretical predictions with high precision differential deuteron dissociation data near zero degrees and obtain a good agreement with experiment over the wide range of targets, from $Z_{t}=6$ to 82 , for which data were measured. The theoretical scheme presented is applicable to halo nucleus systems with similar incident energies/nucleon. Such applications will be presented elsewhere.

\section{Acknowledgments}

The financial support of the United Kingdom Engineering and Physical Sciences Research Council (EPSRC) in the form of Grant No. GR/J95867, of a research scholarship from the Thai Government (for S.R.) and of travel support from the British Council is gratefully acknowledged. The authors would like to express their thanks to Dr Y. Yano, Vice Director RIKEN, and to the RIKEN cyclotron crew for their machine operation. 


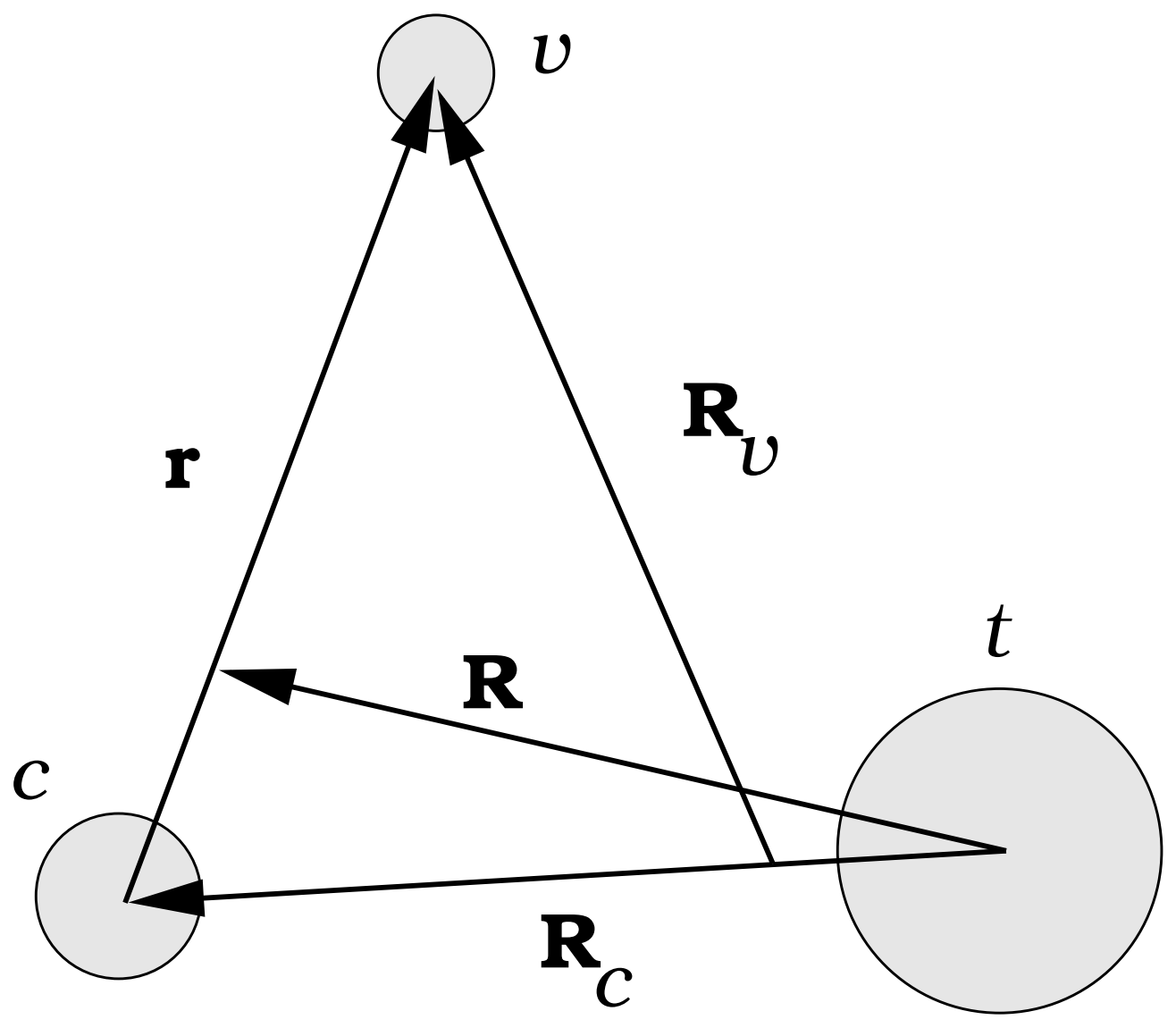

Figure 1

FIG. 1: Definition of the co-ordinate system adopted for the core, valence and target three-body system. 

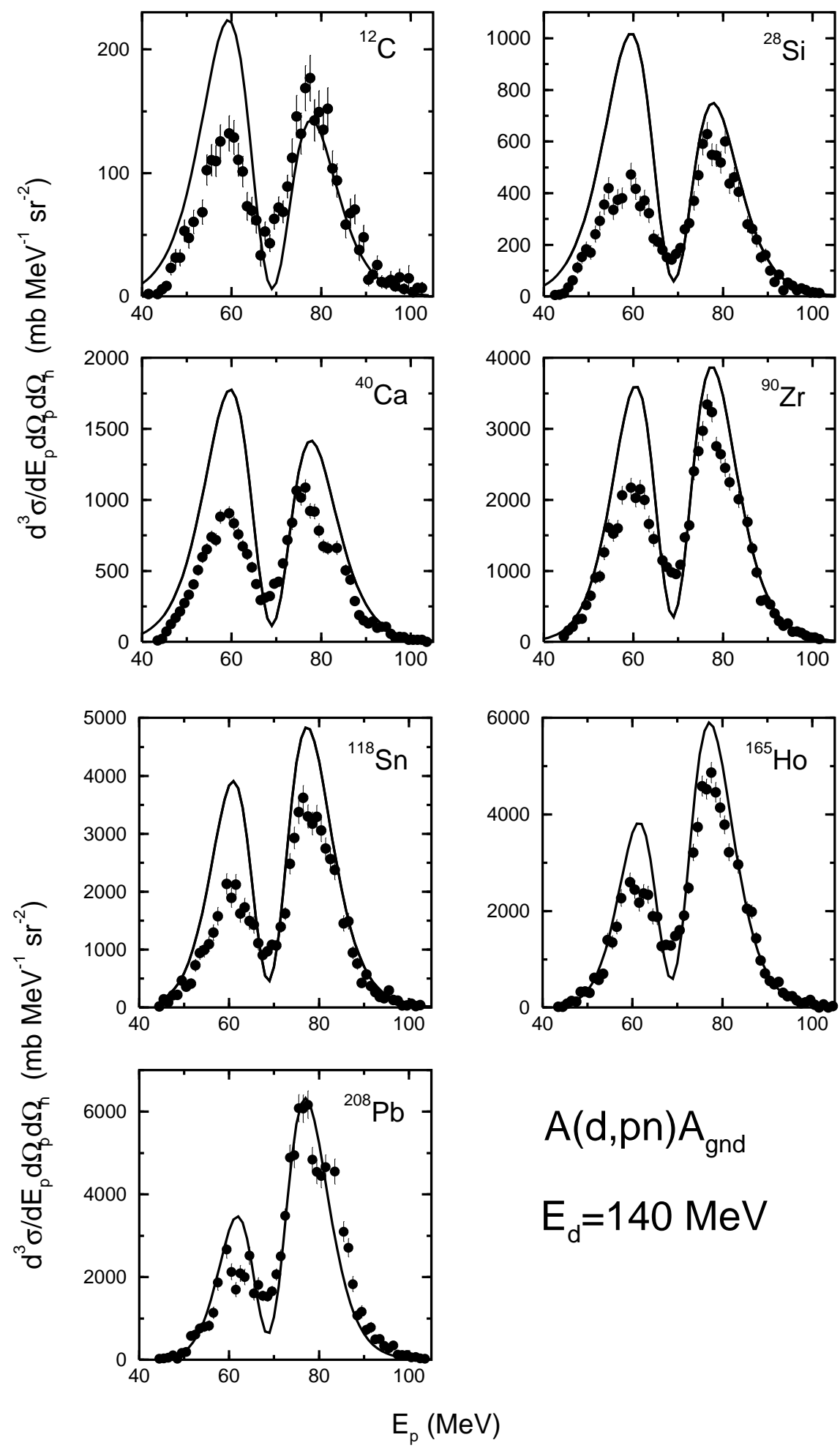

Figure 2

FIG. 2: Experimental and calculated triple differential cross sections for deuteron breakup at $0^{\circ}$ in the laboratory frame at $140 \mathrm{MeV}$. The calculations are averaged over the proton and neutron detection solid angles specified in the text. 